\title{
AVALIAÇÃO DE SISTEMA DE CONTROLE EM REDES INDUSTRIAIS SEM FIO
} ISA $100.11 \mathrm{~A}$

\author{
Sidnei Araújo Júnior*, Heitor Medeiros Florencio ${ }^{\dagger}$, Massao Henrique da Silva*, Daniel \\ Lopes Martins*, Adrião Duarte Dória Neto* \\ ${ }^{*}$ Departamento de Engenharia de Computação e Automação \\ Universidade Federal do Rio Grande do Norte \\ Natal, RN, Brasil \\ †Programa de Pós-Graduação em Engenharia Elétrica e de Computação \\ Universidade Federal do Rio Grande do Norte \\ Natal, RN, Brasil
}

Emails: sidnei.ifrn@gmail.com, heitormflorencio@gmail.com, massaohshen@gmail.com, danlartin@dca.ufrn.br, adriao@dca.ufrn.br

\begin{abstract}
Wireless instrumentation has been used in networked control systems (NCS), generating a control architecture with wireless information traffic. However, there are some features that can influence the design of the control system, such as the packet loss rate or the package delivery rate. This work proposes to evaluate the influence of the packet delivery rate on the variation of the steady state error of a level control. A wireless networked control system architecture with ISA 100.11a technology was implemented to perform the tests and data collection. The results show that there is a direct correlation of packet loss with the controller error.
\end{abstract}

Keywords - Wireless Networked Control Systems, Industrial Wireless Sensor Networks, ISA 100.11a

Resumo- A instrumentação sem fio (wireless) tem sido empregada em sistemas de controle em rede, formando uma arquitetura de controle com o tráfego de informações via meio sem fio. Contudo, existem algumas características que podem influenciar no projeto do sistema de controle, como a taxa de perda de pacotes ou de entrega de pacotes. Neste artigo é proposta a avaliação da influência da taxa de entrega de pacotes na variação do erro de regime permanente de uma malha de controle de nível. Foi implementada uma arquitetura de sistema de controle em rede sem fio com tecnologia ISA 100.11a para realizar os testes e a coleta de dados. Os resultados comprovam que existe uma correlação direta entre o erro do controlador e a perda de pacotes.

Palavras-chave- Sistemas de Controle em Rede, Redes Industriais Sem Fio, ISA 100.11a

\section{Introdução}

Ao analisarmos a linha temporal dos avanços tecnológicos, mais especificamente as três revoluções industriais, é possível observar cada fase que a indústria passou, trazendo consigo inovações em cada um desses períodos. Desde a máquina a vapor até o avanço colossal da microeletrônica, e consequentemente a criação de computadores com maior poder de processamento. Todas essas tecnologias influenciaram a forma de produtividade nos ambientes industriais, que facilitou a automação dos processos.

A concepção de sistemas automatizados que realizam suas tomadas de decisões a partir de uma rede colaborativa de diferentes tecnologias levou a uma nova fase da indústria. Denominada como Industria 4.0 (I4.0) ou Manufatura Avançada, a quarta fase da indústria trás como inovação a interconectividade, reunindo alguns conceitos e tecnologias que formam os pilares de sustentação: Internet das coisas, Segurança cibernética, Computação em nuvem, Manufatura aditiva, Realidade aumentada, Big Data, Robótica colaborativa e Inteligência Artificial (3).

Neste contexto de fusão de tecnologias para garantir maior integração dos sistemas com a finalidade de tomar decisões para otimizar os proces- sos, maximizando lucros e minimizando riscos, a implantação de sistemas de comunicação nos processos de monitoramento e controle deve ser cuidadosamente projetada (7). No cenário atual, é possível observar que grande parte dos sistemas de controle em rede (Networked Control System) é feito de forma cabeada e por mais que esse modelo esteja consolidado no ambiente fabril, algumas necessidades deram espaço às tecnologias sem fio. Não de forma substitutiva, mas como um suplemento ao sistema de conectividade da planta.

Com a inclusão de tecnologias sem fio no sistemas de monitoramento e controle, tornou-se essencial a avaliação do sistemas de controle em redes sem fio (WNSC - Wireless Networked Control System). A tecnologia sem fio oportuniza o controle de variáveis que precisam ser medidas em locais dificilmente acessíveis ao operador. Contudo, a diversidade de interferências eletromagnéticas presentes nas instalações industriais pode afetar diretamente o desempenho das malhas de controle.

Nesse sentido, é fundamental analisar a influência do comportamento da rede sem fio no desempenho do sistema de controle. Dependendo do tipo e da natureza crítica do tráfego de dados da rede (4), um curto período de inconsistência da rede pode causar uma instabilidade no sistema de 
controle.

Neste trabalho, é apresentada a implantação de um sistema de controle em rede sem fio do protocolo ISA 100.11a e avaliada a influência da taxa de perda de pacotes no desempenho do controle realimentado. O desempenho do sistema de controle é mensurado com base na variância do erro em regime permanente.

\section{Sistemas de Controle em Redes Sem Fio}

As tecnologias de comunicação têm modificado a estrutura da clássica pirâmide de automação de processos de manufatura, possibilitando a junção dos diversos sistemas de controle em vários patamares dos processos de fabricação. Para tal abordagem integrativa há o uso dos sistemas de controle em rede, aplicando suas tecnologias e organizações de acordo com o contexto industrial em que a solução será inserida. Segundo Tipsuwan Chow (2003), os NCS podem ser divididos em dois grupos: os que usam estrutura direta e os que usam estrutura hierárquica. Na primeira, os recursos da rede são compartilhados com sensores, atuadores e dispositivos de campo, enquanto que no segundo um controlador principal coordena as operações de controle local sobre a rede. São apresentados nas figuras 1 e 2 respectivamente o modelo de uma estrutura direta e de uma estrutura indireta.

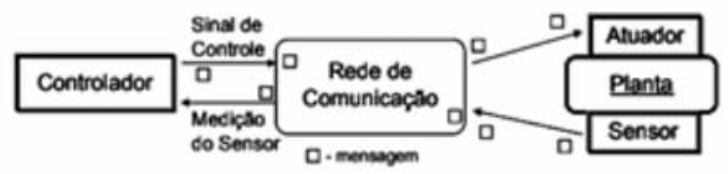

Figura 1: Estrutura direta.

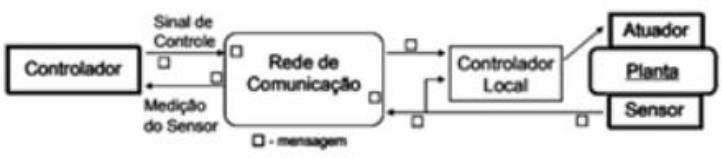

Figura 2: Estrutura hierárquica.

As estruturas de sistemas de controle em redes sem fio seguem o padrão das arquiteturas já apresentadas (direta ou hierárquica), com a ressalva dos elementos utilizados e a mudança do meio de comunicação. Nesses sistemas, a rede de comunicação é baseado no meio sem fio. Os sensores ou atuadores do processo continuam fornecendo e obtendo dados da rede, porém surge um novo elemento que estabelece a comunicação entre a rede e o controlador: o gateway. Esse elemento é responsável pela interface entre a rede sem fio e a rede entre controladores. É ilustrada na figura 3 uma representação da disposição desses elementos.

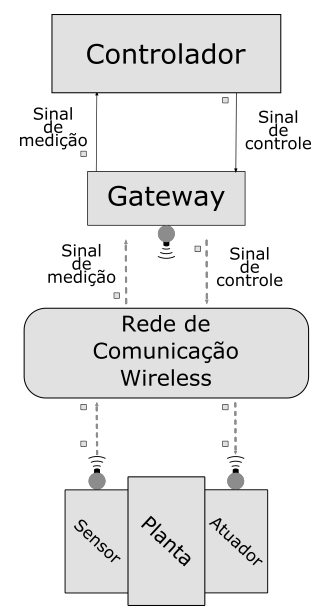

Figura 3: Arquitetura básica de WNCS.

É mostrado na figura 3 que todos os pacotes de medição e de atuação estão trafegando no meio físico sem fio. Enquanto que o controlador do sistema recebe e envia os sinais a partir do gateway, elemento que realiza a interface entre as redes. Além disso, geralmente, o gateway e o gerenciador da rede sem fio estão implementados no mesmo equipamento.

Uma arquitetura alternativa de sistemas de controle em redes sem fio quando não existe atuador com a tecnologia sem fio é apresentada na figura 4 .

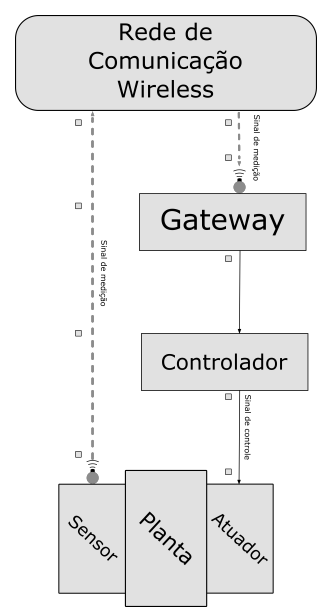

Figura 4: Arquitetura básica de WNCS com controlador local.

Observa-se que na aquitetura apresentada na figura 4 o atuador recebe o sinal de controle direto do controlador através de um meio cabeado. Essa arquitetura será utilizada neste trabalho devido a ausência do atuador com tecnologia sem fio no sistema de controle de nível proposto. 


\subsection{Redes Industriais Sem Fio: ISA 100.11a}

Desenvolvido pela Sociedade Internacional de Automação (ISA) e sendo o primeiro desta família, o padrão ISA100.11a foi desensolvido para aplicações de monitoramento e controle de processos industriais. Um diferencial em relação aos protocolos existentes é o suporte de encapsulamento de dados de protocolos cabeados já difundidos na indústria, como HART, Profibus, Commom Industrial Protocol (CIP), Foundation Fieldbus, Modbus, dentre outros.

Como as aplicações industriais podem envolver sistemas de controle em malha fechada de processos industriais, o protocolo ISA 100.11a define um conjunto de técnicas para garantir a confiabilidade do sistema, a entrega de pacotes em tempo real, a segurança da informação e a interoperabilidade com os demais sistemas (6). Além dos sistemas de controle em malha fechada, existem outras categorias com alto nível de criticidade na implantação, como sistema de segurança e emergência (4).

É apresentada na figura 5 a arquitetura da rede ISA 100.11a.

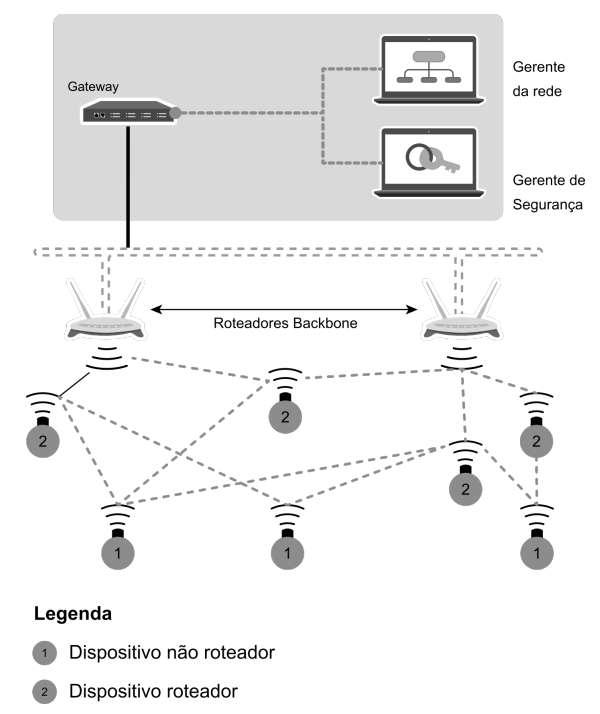

Figura 5: Arquitetura ISA 100.11a.

Costa et al. (2012) descreve a arquitetura do padrão ISA100.11a, que basicamente é constituída de dispositivos de campo roteadores e não roteadores $(\mathrm{I} / \mathrm{O})$, roteadores de backbone, gerenciador de sistema (System Manager), gerenciador de segurança (Security Manager) e o gateway. Os dois gerenciadores e o gateway, normalmente, estão implementados em um único equipamento físico.

O roteador de backbone tem a responsabilidade de fazer o roteamento dos pacotes entre diferentes sub-redes enquanto que o gateway atua com uma interface entre a rede de campo (ISA 100.11a) e a rede de controle. O gerente da rede tem o papel de configurar a comunicação fazendo uma alocação de recursos e escalonamento para gerenciar toda a rede. E o gerente de segurança tem a responsabilidade de gerenciar as políticas de segurança do padrão.

Os nós roteadores executam um grande papel nas redes com topologia em malha, permitindo a troca de dados entre instrumentos que não estão no mesmo raio de alcance de transmissão. A transmissão de um pacote do nó origem até o nó destino pode ser realizada através de múltiplos saltos, ou seja, por vários roteadores. O roteador pode ser utilizado quando um link (conexão) entre dois instrumentos apresenta alta perda de pacote devido ao baixo nível do sinal transmitido. Nesse caso, o instrumento roteador desempenha a função de um caminho alternativo para aumentar a confiabilidade da transmissão.

A relação entre pacotes transmitidos e falhos em um determinado link nas redes industriais sem fio é definida pela taxa de pacotes entregues (PDR - Packet Delivery Rate). A equação 1 define o PDR.

$$
P D R=\left(\frac{T_{x}}{T_{x}+T_{x_{\text {Falhos }}}}\right) * 100 \%
$$

O termo " $T_{x}$ " representa o número de pacotes transmitidos com sucesso e " $T_{x_{\text {Falhos }}}$ " o número de pacotes falhos na transmissão.

Os valores de " $T_{x} " \mathrm{e}$ " $T_{x_{\text {Falhos }}}$ ", geralmente, são acumulados. Com isso, no decorrer do funcionamento da rede, o valor de PDR não consegue sinalizar quando ocorrer falha de pacotes em um curto determinado período de tempo. Essa falha de pacotes, mesmo que em um curto período de tempo, pode ocasionar uma instabilidade no sistema de controle.

Neste trabalho nós utilizamos a taxa de entrega de pacotes instantânea (PDRi - Packet Delivery Rate Instantaneous) para avaliar o desempenho da rede. Neste caso, o PDRi calcula a quantidade de pacotes transmitidos com sucesso e falhos apenas no último instante da coleta.

\subsection{Desempenho do sistema de controle}

Os sistemas de controle em malha fechada geralmente utilizam alguns parâmetros do sistema para avaliação do desempenho, tanto no regime transitório como no permanente. O erro no regime permanente é a característica que predomina as técnicas de avaliação que mostra o quão distante está do valor desejado. Na ação direta, o erro é calculado pela diferença entre a variável de processo (PV) e o setpoint (SP).

O erro quadrático médio (MSE - Mean Squared Error) é uma alternativa quando pretende considerar a tendenciosidade da variável. Além do MSE, outro parâmetro de avaliação é a variância do erro em regime permanente, que consegue detectar uma inconstância no controle. 
A variância do erro calcula a média do quadrado dos desvios padrão dos valores de erro coletados. Contudo, para avaliação do desempenho do controlador em determinados períodos de tempo é indicado o uso da variância móvel do erro (MVE - Moving Variance of Error). O intuito do termo móvel é definir um conjunto de valores, ou janela de valores, que serão utilizados no cálculo da variância. A cada nova amostra o conjunto é atualizado. A equação 2 mostra o cálculo do MVE.

$$
M V E=\frac{\sum_{i=1}^{n}\left(\text { Error }_{i}-\overline{\text { Error }}\right)}{n}
$$

Onde "Error" é um vetor de tamanho $n$ com os valores do erro de regime permanente e "Error" é a média. O vetor "Error" representa a janela móvel de amostras. O parâmetro MVE é utilizado neste trabalho para avaliar o desempenho do sistema de controle quando submetido a uma variação no desempenho da rede.

\section{Metodologia}

O sistema de controle em rede ISA 100.11a implementado neste trabalho segue a arquitetura da figura 6. O processo a ser controlado é o nível de um tanque de $300 \mathrm{~mm}$ que é alimentado por uma bomba que recebe o sinal de $24 \mathrm{~V}$.

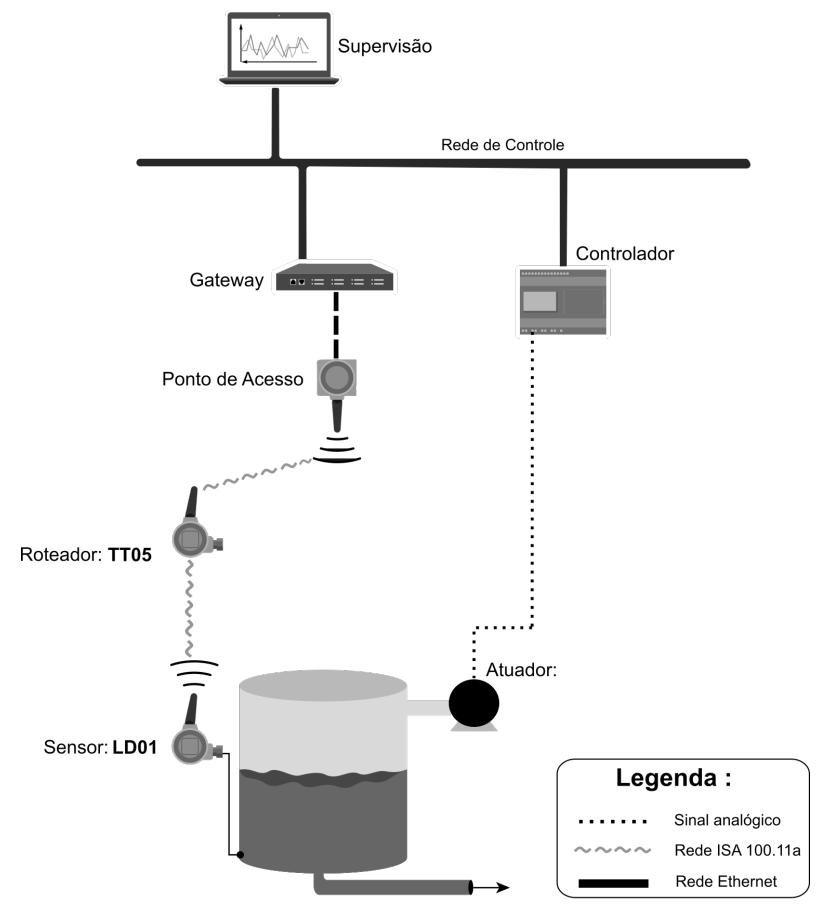

Figura 6: Arquitetura do sistema.

Observa-se na figura 6 que o sinal do sensor de nível (LD01) é enviado via rede ISA 100.11a para um dispositivo roteador que, por sua vez, transmite o pacote para o gerente da rede ISA 100.11a a partir do ponto de acesso. O Controlador comunica-se com o gateway a partir de uma rede de controle do protocolo Modbus TCP. Assim, o sinal da variável de processo (nível) é enviado para o controlador via rede Modbus TCP.

A partir do sinal do nível e do valor desejado (setpoint), o controlador executa o algoritmo do controle PID (Proporcional Integrativo Derivativo) para enviar o sinal da variável manipulada para o atuador (a bomba). A taxa de amostragem do controlador é de 1 segundo. Os ganhos do controle PID foram definidos de acordo com outros testes realizados com o sistema.

Para realizar a medição de nível foi utilizado o sensor de pressão diferencial EJX110B da empresa Yokogawa Electric, tecnologia ISA 100.11a, conforme mostra a figura 7 .

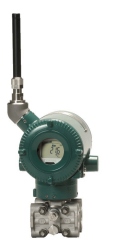

Figura 7: Sensor de pressão diferencial EJX110B. Fonte: (2).

O acesso à rede ISA 100.11a do gerente da rede e do gateway é realizado através do roteador backbone YFGW510 da Yokogawa Electric. Por fim, o gerente da rede, gerente de segurança e o $g a$ teway estão implementados em uma única estação de gerenciamento: YFGW410, conforme mostra a figura 8.

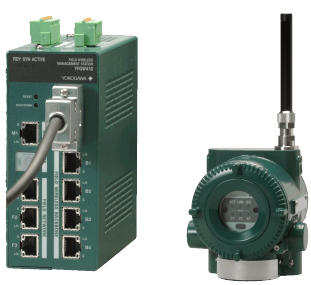

Figura 8: Estação de gerenciamento YFGW410 da Yokogawa Electric e roteador backbone YFGW510. Fonte: (1).

\subsection{Coleta dos dados de avaliação da rede}

Com o intuito de avaliar o desempenho da rede, foram enviados comandos GSAP (Gateway Service Access Point) para comunicação com o gateway da rede. O protocolo GSAP especifica o quadro de comnunicação via Ethernet TCP entre qualquer Gateway de uma rede ISA 100.11a com uma aplicação cliente. Definido na norma ISA 100.11a (IEC 62734), o tipo de serviço definido no comando GSAP estabelece quais são os dados da rede solicitados.

Neste trabalho, o sistema de supervisão utiliza uma ferramenta que envia comandos GSAP para 
solicitar os dados de número de pacotes transmitidos com sucesso e falhos na transmissão de todos os links da rede. A partir dos dados de pacotes transmitidos e falhos é calculado o PDRi (taxa instantânea de pacotes entregues), como é apresentado na equação 1 .

\subsection{Avaliação do controlador}

O controlador PID recebe o setpoint do sistema de supervisão e o valor do nível do gateway para calcular o erro e processar o valor de saída para o atuador. Após o regime transitório do sistema de controle, o sistema estabiliza e gera um erro de regime permanente constante dentro da faixa de aceitação.

Após o sistema alcançar o regime permanente, foi aplicado uma atenuação na antena do dispositivo roteador TT05 para gerar perdas de pacotes nos links entre o sensor de nível LD01 e o ponto de acesso do gerente da rede. As perdas de pacotes gera uma redução no valor do PDRi e, consequentemente, causa uma variação no erro de regime do controlador.

O controlador envia para o sistema de supervisão o valor do erro de regime para que seja calculado a variância móvel do erro (MVE), como mostra a equação 2 . A metodologia proposta neste trabalho avalia o valor do PDRi gerado pela ferramenta de coleta de dados do gateway com o valor do MVE gerado pelo sistema de supervisão para comprovar a forte influência da perda de pacotes no valor da variância do erro (MVE). Essa verificação da influência é indicada pela métrica de correlação.

\section{Resultados e Discussões}

Com a implantação do sistema é possível elaborar os testes de coleta de dados e executar a metodologia de avaliação descrita na seção 3. O objetivo dos testes é estabilizar o controlador em um setpoint fixo, aplicar uma atenuação para causar perda de pacotes nos links e avaliar a correlação entre PDRi e MVE.

Foram realizados três testes com períodos de execução de 30 minutos em média. Todos os três testes seguiram a arquitetura apresentada na figura 6. Desses três testes foram selecionados dois para apresentação de gráficos com os dados.

\subsection{Cenário 01}

Um ponto inicial para ser analisado é o gráfico do PDRi no decorrer deste primeiro cenário de teste. O gráfico da figura 9 apresenta os valores da intensidade do sinal recebido (RSSI - Received Signal Strength Indication) no link de LD01 para TT05 e os valores do PDRi deste link.

Observa-se que do início do teste até aproximadamente $17 \mathrm{~h} 35$ o valor do RSSI manteve-se

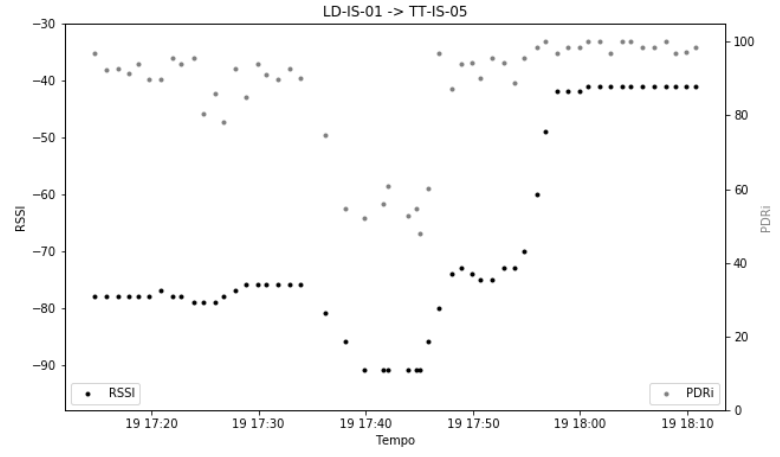

Figura 9: Cenário 01: PDRi versus RSSI.

próximo ao valor de $-80 \mathrm{dBm}$. Enquanto que a variável PDRi manteve-se com pouca perda de pacote, valor aproximado de $90 \%$. Porém, a partir de aproximadamente $17 \mathrm{~h} 35$ foi aplicado a atenuação até $17 \mathrm{~h} 48$.

Neste período de atenuação houve bastante falha de pacote, levando o valor de PDRi para aproximadamente $50 \%$ de entrega de pacotes. A percepção dessas falhas de pacotes estão apresentadas na figura 10, em que estão esboçados os valores oriundos do dispositivo roteador TT05 (variável de processo - PV) na cor preta e os valores reais da medição na cor vermelha.

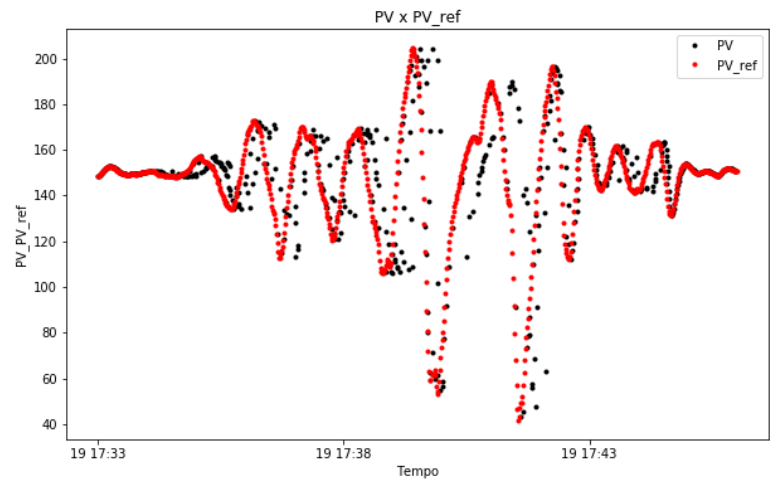

Figura 10: Cenário 01: Análise de falha de pacotes.

Para concepção do gráfico da figura 10 foi inserido outro sensor de nível (LD02) com visada direta para o gateway. Os valores desse outro sensor não sofreram com a atenuação e assim é possível obter os valores reais do nível do tanque, tracejados na cor vermelha na figura 10. Observa-se que no período de atenuação existe uma desigualdade nos valores de medição, isso devido à perda e, consequentemente, retransmissão de aproximadamente $50 \%$ dos pacotes.

A figura 11 apresenta os dados do controlador: medição do nível $(\mathrm{PV})$, setpoint $(\mathrm{SP})$ e saída do controlador (MV).

É possível notar que a saída do PID, variável MV representada no segundo gráfico, apre- 


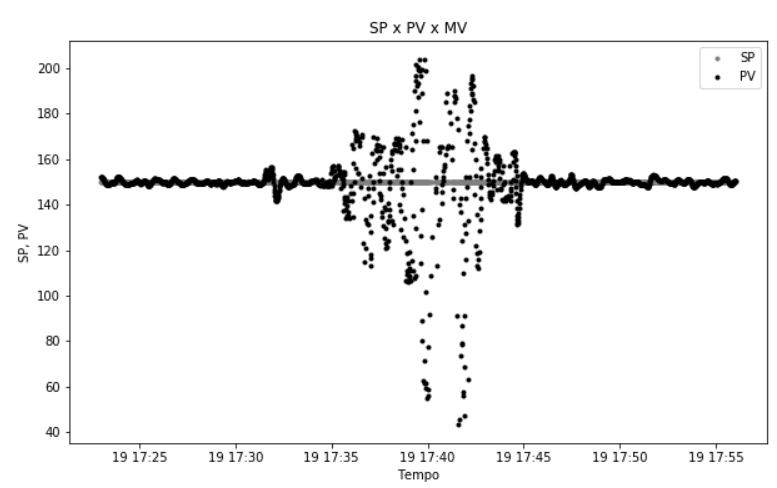

\subsection{Cenário 02}

A atenuação dos links deste segundo teste ocorreu no período entre aproximadamente $14 \mathrm{~h} 05$ e $14 \mathrm{~h} 22$, como mostra a figura 13 .

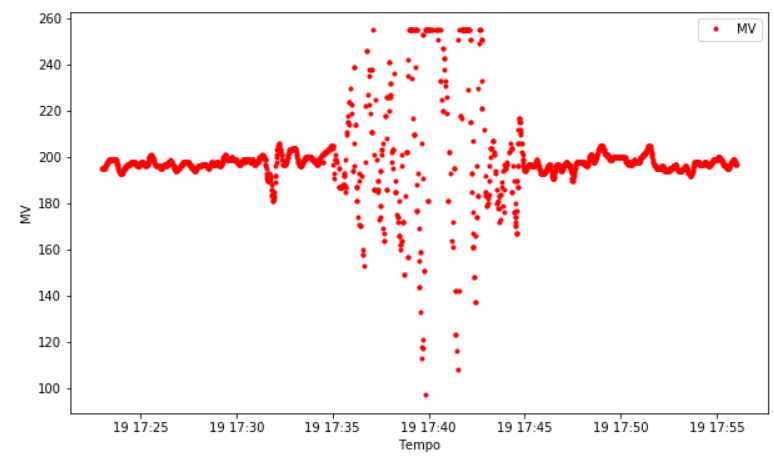

Figura 11: Cenário 01: Variáveis da malha de controle.

senta uma alta variação no período de atenuação. Por consequência, o erro em regime apresenta uma alta variação que é mensurada através do valor de MVE. Os gráficos da figura 12 comprovam visualmente que existe uma influência direta da taxa de perda de pacotes (PDRi) na variação móvel do erro em regime (MVE).
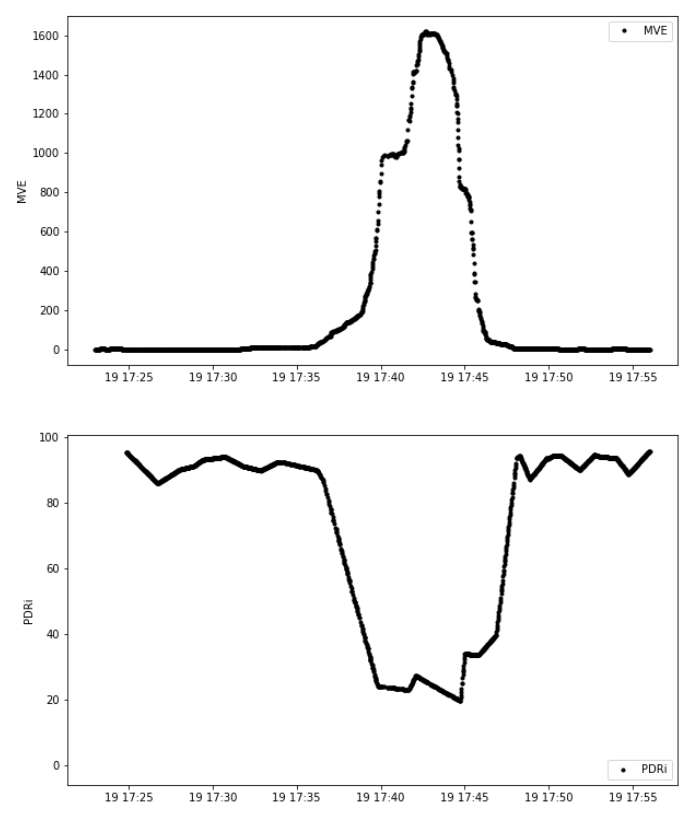

Figura 12: Cenário 01: PDRi versus MVE.

Figura 14: Cenário 02: Variáveis da malha de controle.

A comprovação da influência direta do PDRi na variação móvel do erro de regime permanente do controle é apresentada nos gráficos da figura 15 . Constata-se que uma alta perda de pacotes nas redes ISA 100.11a causa um aumento crescente na variação do erro de regime do sistema de controle. apresentados na figura 14 e corroboram com os resultados apresentados no teste anterior. A perda de pacote também causa uma alta variação no erro de regime permanente do controle.
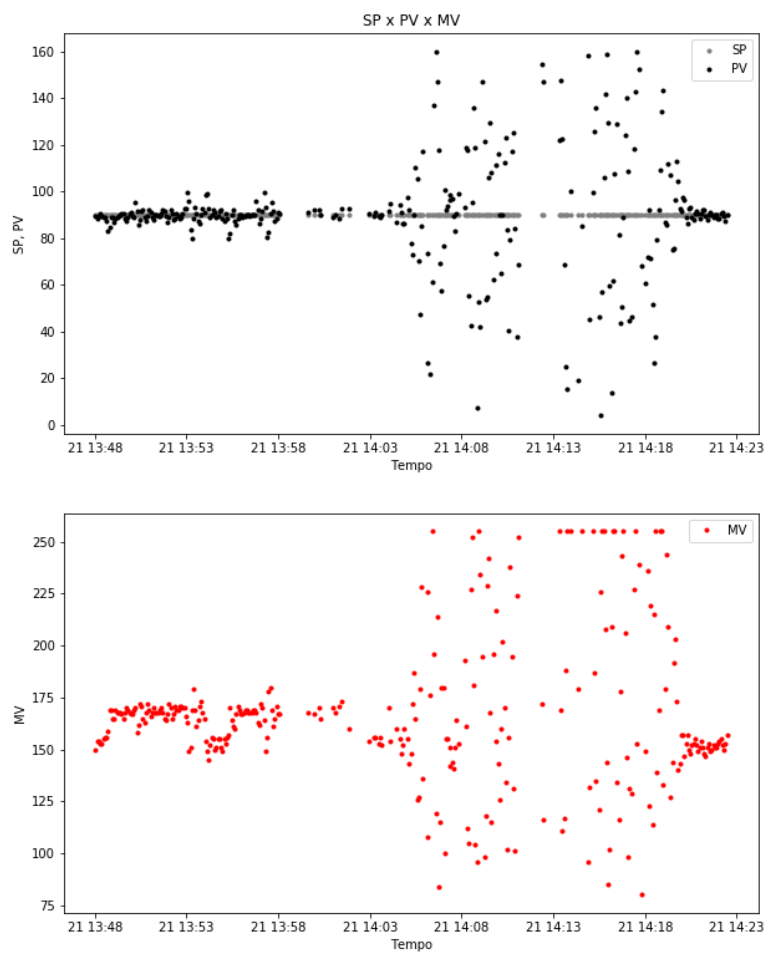

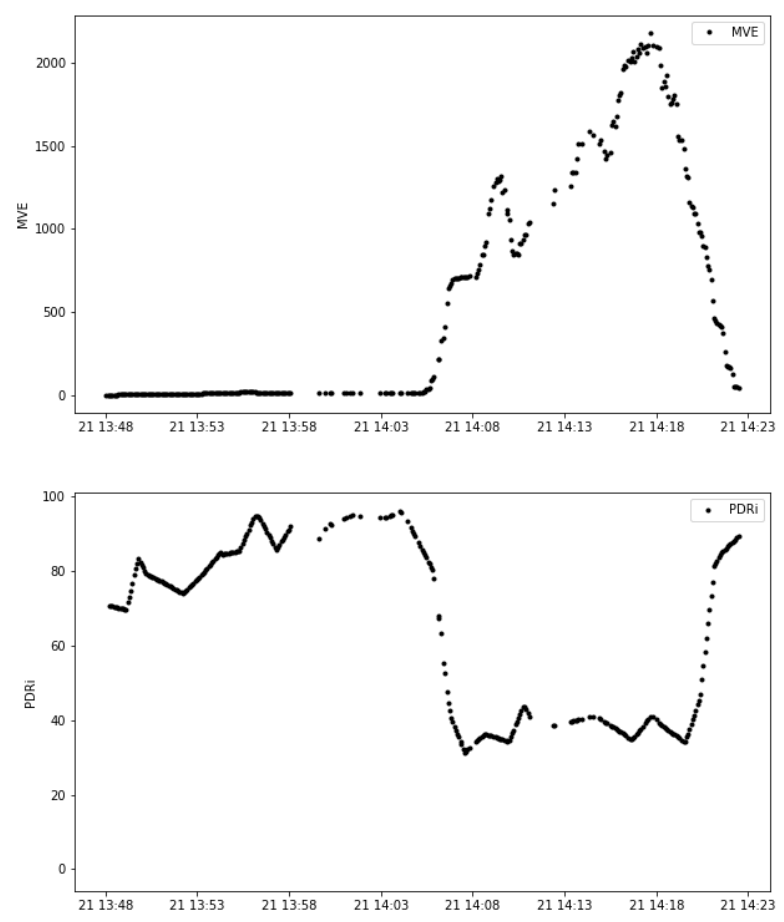

Figura 15: Cenário 02: PDRi versus MVE.

\subsection{Análise de correlação entre PDRi e MVE}

Por fim, após a execução de todos os testes, foram coletados os dados da correlação entre o conjunto de dados do PDRi e o conjunto do MVE de todos os testes. A tabela 1 apresenta a correlação entre as variáveis nos três testes. Essa correlação comprova ainda mais a hipótese de que é possível identificar uma alta variação do erro quando ocorre uma alta perda de pacotes mesmo com os algoritmos de retransmissão.

Tabela 1: Correlação entre PDRi e MVE.

\begin{tabular}{|c|c|}
\hline & Correlação (PDRi, MVE) \\
\hline Teste 01 & -0.719370 \\
\hline Teste 02 & -0.699224 \\
\hline Teste 03 & -0.830738 \\
\hline
\end{tabular}

A média dos três testes apresenta uma correlação de -0.749774, indicando que em aproximadamente $75 \%$ dos dados uma baixa taxa de entrega de pacotes (PDRi) está associada a uma alta variação do erro em regime (MVE).

\section{Contribuições finais}

A tabela de correlação comprova que há influência da perda de pacotes no erro em regime do controlador no sistemas de controle em redes sem fio. Considerando que a implantação de sistemas de controle baseados em comunicação sem fio é uma área recente, desde 2010, estudos de avaliação da influência do desempenho da rede no comportamento das malhas de controle podem direcionar os projetistas quanto ao uso das tecnologias.

Considerando que é possível saber a faixa de variação do erro em regime quando existe uma determinada atenuação no ambiente, como mostra a metodologia deste trabalho, a equipe de projeto pode julgar se é viável a implantação do sistema. Além disso, os dados resultantes da metodologia fornecem uma base para inferência de possíveis falhas do controlador com o uso de técnicas de predição.

Portanto, este trabalho apresentou resultados que avaliam o desempenho de um sistema de controle de nível em rede sem fio ISA 100.11a e mostra-se uma excelente ferramenta para uso em projetos e predição.

\section{Agradecimentos}

Ao $\mathrm{CNPq}$ pelo auxílio financeiro.

\section{Referências}

[1] YOKOGAWA Electric. YFGW510 Field Wireless Access Point. Available from: https://www.yokogawa.com/solutions/productsplatforms/field-instruments/fieldwireless/wireless-infrastructure/yfgw510field-wireless-access-point/. Access Apr 29, 2019.

\section{[2] YOKOGAWA Electric. EJX110B Wireless Differential Pressure Transmitter. Available from:} https://www.yokogawa.com/solutions/productsplatforms/field-instruments/fieldwireless/wireless-differential-pressure-pressuretransmitters/ejx110b-wireless-differentialpressure-pressure-transmitter/. Access Apr 29, 2019 .

[3] SCHWAB, Klaus. The Fourth Industrial Revolution. Genebra: World Economic Forum, 2016.

[4] RAZA, Mohsin et al. A critical analysis of research potential, challenges, and future directives in industrial wireless sensor networks. IEEE Communications Surveys Tutorials, v. 20, n. 1, p. 39-95, 2017.

[5] TIPSUWAN, Yodyium; CHOW, Mo-Yuen. Control methodologies in networked control systems. Control engineering practice, v. 11, n. 10, p. 1099-1111, 2003.

[6] MARTINS, D. L. ; FLORENCIO, H. M. , et al. Cenário Atual das Redes Industriais Sem Fio: Experimentos e Testes de Campo. In: Rio Automação 2015, 2015, Rio de Janeiro. Rio Automação 2015, 2015.

[7] ZIPPEL, S. Process Industry 4.0. Revista InTech, 2018. 